Special issue of the 3rd International Conference on Computational and Experimental Science and Engineering (ICCESEN 2016)

\title{
Adult Patient Radiation Doses with Multislice Computed Tomography Exam: MSCT Standard Protocols
}

\author{
U. KARA ${ }^{a, *}, \mathrm{~A} \mathrm{KAYA}^{b}$, H.O. TEKIN ${ }^{c}$ AND İ. AKKURT ${ }^{d}$ \\ ${ }^{a}$ Süleyman Demirel Üniversity, Vocational School of Healt Services, 32200, Isparta, Turkey \\ ${ }^{b}$ Süleyman Demirel Üniversity, Science and Applied Institutes, Isparta, Turkey \\ ${ }^{c}$ Usküdar University, Vocational School of Health Services, Radiotherapy Department, Istanbul, Turkey \\ ${ }^{d}$ Süleyman Demirel Üniversity, Science and Arts Faculty, Physics Department, 32200, Isparta, Turkey \\ Radiation is energy, and it is widely used in a variety of fields, especially in industry and medical science. \\ In hospital, ionizing radiation like X-ray is an extensive exam that has been used to help physicians to have a view \\ into the body, without having to make a medical application. Computed tomography scan uses ionizing radiation, \\ and it is a nearly perfect diagnostic unit that allows the physician to see the picture of the human body. Computed \\ tomography scan technology has progressed over the years, and it is an increasingly powerful and effective unit in \\ the diagnostic radiology. Exposure to ionizing radiation is known to increase the risk of cancer. The aim of this \\ study was to assess the radiation exposure received during computed tomography in a sample representative of the \\ current state of practice in adult patients.
}

DOI: 10.12693/APhysPolA.132.1126

PACS/topics: 87.50.yk, 87.57.uk

\section{Introduction}

Radiation is widely used in a variety of fields, especially at the medical centers. We know that clinical radiation is necessary for diagnosis and we use it in medical centers. Radiology, nuclear medicine, radiation oncology clinics use radiation for diagnosis and treatment. Radiology departments often use ionizing radiation for diagnosis.

Computed tomography (CT) is a medical imaging technology in radiology facilities, which has been used for screening and diagnosis of medical conditions. CT involves taking numerous X-ray images of a body area or organ, and these images are reconstituted into computer-generated pictures $[1,2]$. A large series of twodimensional X-ray images (slices) of the inside of an object are taken around a single axis of rotation.

$\mathrm{CT}$ has transformed much of medical imaging by providing three-dimensional views of the organ or body region of interest. The use of CT has increased rapidly, both in the United States and elsewhere, notably in Japan. According to a survey conducted in 1996, the number of CT scanners per 1 million population was 26 in the United States and 64 in Japan. It is estimated that more than 62 million CT scans are currently made each year in the United States, as compared to about 3 million in $1980[3,4]$.

\section{Materials and methods}

In this study, we have worked in the radiology department in a hospital, which uses multi-slice computed tomography (CT/128 slice) medical imaging unit (Fig. 1).

\footnotetext{
*corresponding author; e-mail: umitkara@sdu.edu.tr
}

We measured radiation doses of radiology patients during multi-slice computed tomography (MSCT). We recorded radiation factors and radiation treatments in a conventional protocol (head) during radiology examination using multi-slice computed tomography.
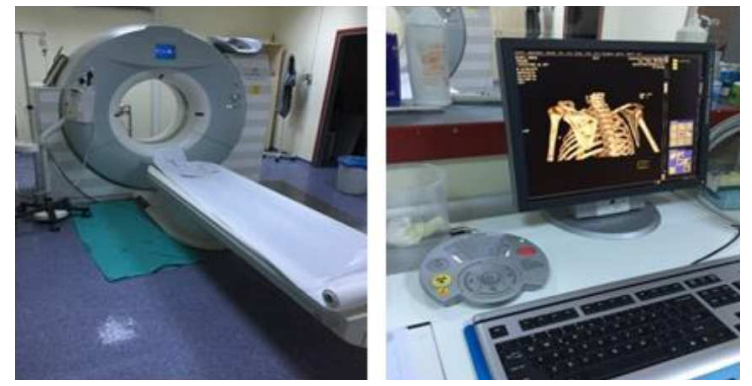

Fig. 1. Multi slice computed tomography unit used in experiments.

In this study, we measured radiation factors and radiation doses for the MSCT, unit, which is most widely used X-ray imaging unit in radiology. When we have measured the doses, patients and medical staff were in routine locations. Head scan protocol, which is routinely used during CT, was employed in this study. The IAEA definitions were adopted for the dosimetric quantities, expressed regarding Kerma [5]. The weighted air Kerma index $C_{\mathrm{W}}$ (Eq. (1)) has the objective of measuring the air kerma index within the phantoms. The volumetric air Kerma index $C_{\text {Vol }}$ (Eq. (2)) provides the estimate of the dose in a single section. The air Kerma-length product (PKL, Ct) (Eq. (3)) provides the air Kerma in the whole irradiated area, during the acquisition of the tomographic image. We have also adopted the concept of effective dose estimated from (PKL,Ct), as a function of a conversion factor $k$ (Eq. (4); Table I), depending only 
on the irradiated body region [6]. The effective dose was found as Dose Length Product $\times$ Conversion Factor. We have repeated these measurements in a radiology department for one month, using Polimaster DI-1621 X-ray and gamma ray radiation personal dosimeter.

$$
\begin{aligned}
& C_{\mathrm{W}}=1 / 3\left(C_{\mathrm{PMMA}, 100, \mathrm{C}}+2 C_{\mathrm{PMMA}, 100, \mathrm{P}}\right), \\
& C_{\mathrm{Vol}}=C_{\mathrm{W}} / \text { pitch, } \\
& \mathrm{PKL}, \mathrm{Ct}=\sum N c \mathrm{Vol} j j P I t, \\
& E=k \times \mathrm{PKl}, \mathrm{Ct}, \\
& E=\mathrm{DLP} \times k .
\end{aligned}
$$

TABLE I

Conversion factor for calculations of effective doses [3].

\begin{tabular}{c|c|c|c|c|c}
\hline \hline $\begin{array}{c}\text { Body } \\
\text { region }\end{array}$ & $\begin{array}{c}0 \\
\text { years-old }\end{array}$ & $\begin{array}{c}1 \\
\text { year-old }\end{array}$ & $\begin{array}{c}5 \\
\text { years-old }\end{array}$ & $\begin{array}{c}10 \\
\text { years-old }\end{array}$ & Adult \\
\hline $\begin{array}{c}\text { Head } \\
\text { and neck }\end{array}$ & 0.013 & 0.0085 & 0.0057 & 0.0045 & 0.0031 \\
Head & 0.011 & 0.0067 & 0.0040 & 0.0032 & 0.0021 \\
Neck & 0.017 & 0.012 & 0.011 & 0.0079 & 0.0059 \\
Chest & 0.039 & 0.026 & 0.018 & 0.013 & 0.014 \\
Abdomen & 0.049 & 0.030 & 0.020 & 0.015 & 0.015 \\
Trunk & 0.044 & 0.028 & 0.019 & 0.014 & 0.015
\end{tabular}

\section{Results and discussion}

The meaning of CT dose index (CTDI). CT is a standardized measure of radiation dose output of a CT scanner, which allows the user to compare radiation output of different CT scanners. CTDIvol is obtained by dividing CTDIw by pitch factor. DLP meaning of Dose Length Product. DLP is the product of the CTDIvol and the scan length for a group of scans. This number can be summed over the entire exam, to give an estimate of the total dose. The value is expressed in $m G y \mathrm{~cm}$. Pitch is defined as the ratio of the table travel per rotation to the full nominal beam width [7-9].

Figure 2 shows the organ doses, sufficient doses, gender and risk indices in adult $\mathrm{CT}$.

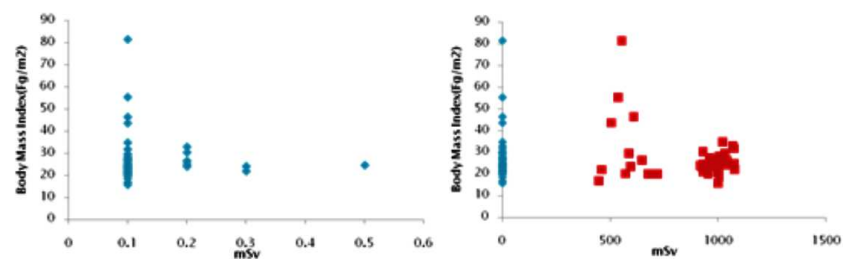

Fig. 2. Body mass index vs radiation dose.

Multi-slice CT is an important diagnostic imaging unit in medicine. Multi-slice CT units have become safer over the years, but due to their increasing usage and frequency of scans, radiation exposure is a significant public health concern. In most circumstances, the risk to an individual patient of developing a malignant tumor because of CT is small and acceptable, compared to the substantial benefit, although there is a large uncertainty in risk estimates at these dose levels. Usage of CT has increased over the past several years. Number of CT examinations has also increased, in part due to the increased speed of image acquisition, allowing vascular, cardiac, and multiphase examinations, all associated with higher doses. Thus, greater use of CT has resulted in a concomitant increase in the medical exposure to ionizing radiation [9-11].

\section{Acknowledgments}

This work has been supported by Suleyman Demirel University Foundation Unit (BAP) with the project number of 4898 YL2 17.

\section{References}

[1] Health Canada, Whole body screening using MRI or CT technology, Ottawa 2003.

[2] H.O. Tekin, U. Kara, J. Communication Comput. 13, 32 (2016).

[3] United Nations Scientific Committee on the Effects of Atomic Radiation, Sources and effects of ionizing radiation, UNSCEAR 2000 report to the General Assembly, New York 2000.

[4] IMV 2006 CT Market Summary Report, Des Plains, IL: IMV Medical Information Division, 2006.

[5] International Atomic Energy Agency, Dosimetry in diagnostic radiology: an international code of practice, Technical Reports Series no. 457 , IAEA, Vienna 2007.

[6] American Association of Physicists in Medicine, The measurement, reporting, and management of radiation dose in CT, AAPM Report No. 96, AAPM, College Park 2008.

[7] C.H. McCollough, F.E. Zink, Med. Phys. 26, 2223 (1999).

[8] International Electrotechnical Commission (IEC), Medical Electrical Equipment. Part 2-44: Particular requirements for the safety of $x$-ray equipment for computed tomography, IEC publication No. 60601-244. Ed. 2.1, International Electrotechnical Commission (IEC), Geneva 2002.

[9] E.S. Amis, P.F. Butler, K.E. Applegate, S.B. Birnbaum, L.F. Brateman, J.M. Hevezi, F.A. Mettler, R.L. Morin, M.J. Pentecost, G.G. Smith, K.J. Strauss, R.K. Zeman, J. Am. College Radiol. 4, 272 (2007)

[10] National Council on Radiation Protection and Measurements, Ionizing Radiation Exposure of the Population of the United States, NCRP report 160, www.ncrponline.org/, accessed October 7, 2009.

[11] H.O. Tekin, T. Manici, C. Ekmekci, J. Health Sci. 4, 131 (2016). 